\title{
Photoionization dynamics and angular squeezing phenomenon in intense long-wavelength laser fields
}

\author{
Zhongyuan Zhou ${ }^{1, *}$ and Shih-I Chu ${ }^{1,2, \dagger}$ \\ ${ }^{1}$ Department of Chemistry, University of Kansas, Lawrence, Kansas 66045, USA \\ ${ }^{2}$ Center for Quantum Science and Engineering, Department of Physics, National Taiwan University, Taipei 10617, Taiwan
}

(Received 19 April 2010; published 11 March 2011)

\begin{abstract}
We develop a coherent-state Ehrenfest trajectory (CSET) approach for the nonperturbative study of full electronic and nuclear dynamics of molecules interacting with intense laser fields. In this approach, electrons and nuclei are characterized by CSETs, dynamic processes are identified by the properties of the CSETs, and the transition probability of a process is calculated from the number of CSETs in this process. We apply this approach to simulate the full dynamics of $\mathrm{H}_{2}$ in intense linearly polarized laser fields. In the tunneling regime, the photoelectron energy spectra show a pronounced low-energy structure (LES) and the predicted scaling law of the LES with respect to the Keldysh parameter is in very good agreement with that observed in the latest experiments. Moreover, the photoelectron angular distribution is found to be squeezed along the laser field direction with the increase of laser wavelength and/or intensity.
\end{abstract}

DOI: 10.1103/PhysRevA.83.033406

PACS number(s): $33.80 . \mathrm{Rv}, 33.80 . \mathrm{Eh}, 42.50 . \mathrm{Hz}$

\section{INTRODUCTION}

Recent experiments [1,2] show that in the tunneling ionization regime when Keldysh parameter $\gamma=\sqrt{I_{P} / 2 U_{P}}<1$ the photoelectron energy spectra (PESa) of atoms and molecules in long-wavelength intense laser fields display some new features in the low-energy region that cannot be explained by the existing tunneling-ionization models such as the KeldyshFaisal-Reiss model [3-5], where $I_{P}$ is the field-free ionization potential and $U_{P}=e^{2} \varepsilon_{0}^{2} / 4 m \omega^{2}$ is the ponderomotive energy (here, $\varepsilon_{0}$ and $\omega$ are the amplitude and angular frequency of the laser field, and $e$ and $m$ are the charge and mass of the electron, respectively). These features include [1]: (a) The PESa have an unexpected pronounced low-energy structure (LES) that can extend up to quite high energy when $\gamma \ll 1$; (b) the width of the LES increases with the decrease of $\gamma$ in the light of scaling law $\gamma^{-(1.78 \pm 0.1)}$; and (c) the LES and the scaling law are universal. Although qualitative exploration to the first feature has been made for the noble gas atoms using the semiclassical method [2] the quantitative investigation to these features is still unavailable. Moreover, the new features are achieved primarily from the experimental results of atoms. Thus it is essential to examine these features for molecules so as to explore their universality. One of the goals of this work is to quantitatively investigate these features for molecules through the photoionization of $\mathrm{H}_{2}$ in intense long-wavelength laser fields.

For molecules in long-wavelength laser fields, nuclear relaxation becomes significant and dynamics study involves twelve-dimensional (12D) calculation even for the simplest $\mathrm{H}_{2}$ molecule if nuclear motions are taken into account. However, within the current capability of supercomputers the conventional fully $a b$ initio methods based on the solution of the time-dependent Schrödinger equation (TDSE) are only limited to six-dimensional (6D) systems. Accurate $a b$ initio

\footnotetext{
*zyzhou@ku.edu

†sichu@ku.edu
}

calculations, such as high harmonic generation [6] and double photoionization [7-9], etc., for two-electron quantum systems have been accomplished only recently. Direct solution of TDSE for the systems beyond 6D remains a major computational challenge at the current time. Thus it is desirable to develop new theoretical and computational methodologies to explore the full electronic and nuclear dynamics of molecules in intense laser fields.

The computational challenge mainly comes from the fact that the total number of basis functions (grid points) $\mathcal{N}$ in the conventional TDSE methods typically increases exponentially with the dimension of system $\mathcal{M}$ as $\mathcal{N} \sim \mathcal{K}^{\mathcal{M}}$, where $\mathcal{K}$ is the number of basis functions (grid points) for each dimension. In principle, this obstacle can be conquered simply by decreasing $\mathcal{N}$. One kind of method to reduce $\mathcal{N}$ is to decrease $\mathcal{K}$ by using optimized basis functions [10]. One typical example of this method is multiconfiguration time-dependent Hartree method that uses time-dependent single-particle functions as the basis set $[11,12]$. This kind of method can indeed reduce $\mathcal{N}$ but it is still suffering from the exponentially scaling difficulty [13]. This difficulty becomes more aggravated for the electron dynamics in intense laser fields because the large amplitude of electronic motion makes it impossible to use small $\mathcal{K}$ [14]. Another kind of method to reduce $\mathcal{N}$ is to change the relation between $\mathcal{N}$ and $\mathcal{M}$ by developing novel approaches. One such approach is the coupled coherent state (CCS) method that makes use of time-dependent coherent states (CSs) as the basis set $[10,15-17]$. In the CCS method, $\mathcal{N}$ is greatly reduced because it scales up quadratically with $\mathcal{M}$ as $\mathcal{N} \sim \mathcal{M}^{2}$. The CCS method has been successfully applied to a number of multidimensional calculations [10,14-18].

In this work, we develop a more feasible coherent-state Ehrenfest trajectory (CSET) approach for the study of full dynamics of molecules in intense laser fields based on the coherent-state trajectories [17] that are determined by coupled Ehrenfest equations. In this approach, the electrons and nuclei in real space are mapped to the phase space. Instead of wave functions the electrons and nuclei are characterized by a group of quantum trajectories-CSETs in phase space. 
The dynamic processes are identified by the properties of the CSETs and the transition probability of a process is calculated from the number of CSETs in this process. The computational cost of this approach is linearly proportional to $\mathcal{M}$ and thus it can be applied to large molecules. As an application, the approach is used to simulate the full dynamics of $\mathrm{H}_{2}$ in laser fields and study energy-angular distribution of photoelectrons through second-order differential ionization probability (SDIP). Particularly, the wavelength and intensity dependence of the PESa of $\mathrm{H}_{2}$ in long-wavelength intense laser fields are investigated to examine the universality of the LES and the scaling law of the LES observed for atoms in the recent experiments. The wavelength and intensity dependence of photoelectron angular distribution (PAD) of $\mathrm{H}_{2}$ in laser fields are also explored in order to have a further insight into the tunneling photoionization. We find that the PAD is squeezed with the increase of laser wavelength and/or intensity, and thus the photoelectrons focus on the direction of the laser field at long-wavelength and/or high-intensity limits.

\section{THEORETICAL METHODOLOGY}

\section{A. Coherent-state Ehrenfest trajectory}

For a generic system with $N_{p}$ particles, the time-dependent wave function $|\Psi(t)\rangle$ can be expanded in time-dependent basis states (TDBSs) $\left\{\left|\psi_{n}(t)\right\rangle \mid n=1,2, \ldots\right\}$ as

$$
|\Psi(t)\rangle=\sum_{n=1}^{N_{t}} c_{n}(t)\left|\psi_{n}(t)\right\rangle
$$

where $N_{t}$ is the total number of TDBSs and $c_{n}(t)$ is the expansion coefficient. The TDBSs can be constructed by any kind of basis functions, such as time-dependent singleparticle wave functions [11,12] and time-dependent Gaussian functions $[15,16]$, etc. In this work, we construct $\left|\psi_{n}(t)\right\rangle$ in terms of $N_{p}$ three-dimensional (3D) CSs $\left\{\left|\chi_{n}(j)\right\rangle, j=\right.$ $\left.1,2, \ldots, N_{p}\right\}$ as $\left|\psi_{n}(t)\right\rangle=\left|\chi_{n}(1)\right\rangle\left|\chi_{n}(2)\right\rangle \cdots\left|\chi_{n}\left(N_{p}\right)\right\rangle$, as in the CCS method [14-18]. In coordinate space, the 3D CS of the $j$ th particle in the $n$th TDBS, $\left\langle\mathbf{x} \mid \chi_{n}(j)\right\rangle$, is given by $[14,18]$

$$
\begin{aligned}
\left\langle\mathbf{x} \mid \chi_{n}(j)\right\rangle= & \frac{1}{\left(2 \pi \sigma_{j n}^{2}\right)^{3 / 4}} \exp \left[-\frac{1}{4 \sigma_{j n}^{2}}\left(\mathbf{x}-\mathbf{q}_{j n}\right)^{2}\right. \\
& \left.+\frac{i}{\hbar} \mathbf{p}_{j n} \cdot\left(\mathbf{x}-\mathbf{q}_{j n}\right)+\frac{i}{2 \hbar} \mathbf{p}_{j n} \cdot \mathbf{q}_{j n}\right]
\end{aligned}
$$

where $\mathbf{q}_{j n}$ and $\mathbf{p}_{j n}$ are the position and momentum of the CS wave packet, $\sigma_{j n}=\sqrt{\hbar / 2 m_{j} \varpi_{j n}}$ is the width of the wave packet, $m_{j}$ is the mass of the particle, and $\varpi_{j n}$ is the frequency parameter. In principle, $\varpi_{j n}$ can be used to adjust the width and optimize the quality of the CS during the propagation. However, frozen wave packets with fixed widths are more robust in numerical calculation $[13,17]$. Therefore in our calculations we employ the frozen CSs and take $\varpi_{j n}=1[15,16]$. The CSs are normalized but nonorthogonal. The overlap integral of any two 3D CSs is given by

$$
\begin{aligned}
s_{n n^{\prime}}(j) & =\left\langle\chi_{n}(j) \mid \chi_{n^{\prime}}(j)\right\rangle \\
& =\exp \left(\mathbf{z}_{j n}^{*} \cdot \mathbf{z}_{j n^{\prime}}-\frac{\left|\mathbf{z}_{j n}\right|^{2}}{2}-\frac{\left|\mathbf{z}_{j n^{\prime}}\right|^{2}}{2}\right),
\end{aligned}
$$

where $z_{j n}=q_{j n} / 2 \sigma_{j n}+i \sigma_{j n} p_{j n} / \hbar$. Obviously, $s_{n n^{\prime}}(j)=1$ when $z_{j n}=z_{j n^{\prime}}$ and $s_{n n^{\prime}}(j)$ is very small when $z_{j n}$ is far from $z_{j n^{\prime}}$. The identity operator of the CS is given by

$$
\widehat{I}=\frac{1}{(2 \pi \hbar)^{3}} \int\left|\chi_{n}(j)\right\rangle\left\langle\chi_{n}(j)\right| d \mathbf{p}_{j n} d \mathbf{q}_{j n} .
$$

In phase space, the CS is localized and centered at the point $\left(\mathbf{q}_{j n}, \mathbf{p}_{j n}\right)$, and thus its position can be represented by $z_{j n}=\left\{\mathbf{q}_{j n}, \mathbf{p}_{j n}\right\}$. Evolution of $z_{j n}$ is governed by a group of generalized Ehrenfest equations as shown below and constitutes a trajectory which we call CSET. Since the CS is uniquely determined by its CSET, the TDBS is uniquely characterized by a CSET vector of the $N_{p}$ particles $\mathbf{Z}_{n}=$ $\left(\mathbf{z}_{1 n}, \mathbf{z}_{2 n}, \ldots, \mathbf{z}_{N_{p} n}\right)^{T}$ and the system by an ensemble of $N_{t}$ CSET vectors $\left\{\mathbf{Z}_{1}, \mathbf{Z}_{2}, \ldots, \mathbf{Z}_{N_{t}}\right\}$. In the ensemble, the CSETs in different TDBSs are completely different from each other. With the help of Eq. (3), when the dimension of the system $N_{d}=3 N_{p} \rightarrow \infty$, the overlap integral of any two TDBSs $S_{n n^{\prime}}=\prod_{j=1}^{N_{P}} s_{n n^{\prime}}(j)$ satisfies

$$
S_{n n^{\prime}}=\delta_{n n^{\prime}}
$$

Thus the TDBSs constructed in terms of CSs are orthonormalized for the system with an infinite number of particles. For a system with a large number of particles, Eq. (5) offers a condition for the most probable event of the system. For molecular systems, $N_{d} \geqslant 12\left(N_{d}=12\right.$ for the simplest $\left.\mathrm{H}_{2}\right)$ and the most probable probability obtained by using this condition provides a good description of the processes of the systems as shown below.

\section{B. Ehrenfest equation for the coherent-state Ehrenfest trajectories}

In general, both $c_{n}(t)$ and $\mathbf{Z}_{n}(t)$ are time dependent and coupled together during the propagation. The general form of coupled equations can be derived using the quantum time-dependent variational principle. These equations are very difficult to solve for the system with many particles [17]. A more feasible method is to optimize one of $c_{n}(t)$ and $\mathbf{Z}_{n}(t)$ using the variational principle after the other is reasonably chosen prior. One option is to choose $\mathbf{Z}_{n}(t)$ from the Hamilton canonical equation and optimize $c_{n}(t)$. This will give the equations of the CCS method [16,17]. Another alternative option is to choose $c_{n}(t)$ and apply the variational principle to $Z_{n}(t)$. For the system described by the wave function of Eq. (1) and the TDBSs constructed in terms of CSs, the Lagrangian is 
given by

$$
\begin{aligned}
\mathcal{L}= & \left\langle\Psi(t)\left|i \hbar \frac{\partial}{\partial t}-H(t)\right| \Psi(t)\right\rangle=\sum_{m, n}\left\{\frac{i \hbar}{2}\left[c_{m}^{*} \dot{c}_{n}-\dot{c}_{m}^{*} c_{n}\right] S_{m n}+\frac{i \hbar}{2}\left[\left(\left(\mathbf{Z}_{m}^{\dagger}-\mathbf{Z}_{n}^{\dagger}\right) \cdot \dot{\mathbf{Z}}_{n}+\frac{\mathbf{Z}_{n}^{\dagger} \cdot \dot{\mathbf{Z}}_{n}}{2}-\frac{\dot{\mathbf{Z}}_{n}^{\dagger} \cdot \mathbf{Z}_{n}}{2}\right)\right.\right. \\
& \left.\left.-\left(\dot{\mathbf{Z}}_{m}^{\dagger} \cdot\left(\mathbf{Z}_{n}-\mathbf{Z}_{m}\right)+\frac{\dot{\mathbf{Z}}_{m}^{\dagger} \cdot \mathbf{Z}_{m}}{2}-\frac{\mathbf{Z}_{m}^{\dagger} \cdot \dot{\mathbf{Z}}_{m}}{2}\right)\right] c_{m}^{*} c_{n} S_{m n}-c_{m}^{*} c_{n} S_{m n} H_{\mathrm{ord}}\left(\mathbf{Z}_{m}^{\dagger}, \mathbf{Z}_{n}\right)\right\}
\end{aligned}
$$

where $H(t)$ is the Hamiltonian of the system, $\dot{c}_{n}=d c_{n} / d t$, $\dot{\mathbf{Z}}_{n}=d \mathbf{Z}_{n} / d t$, the asterisk and dagger represent the complex conjugate and complex conjugate transpose, respectively, and $H_{\text {ord }}\left(\mathbf{Z}_{m}^{\dagger}, \mathbf{Z}_{n}\right)$ is the ordered Hamiltonian. The ordered Hamiltonian is introduced in the calculation of the Hamiltonian matrix element $\left\langle\psi_{m}|H| \psi_{n}\right\rangle=H_{\text {ord }}\left(\mathbf{Z}_{m}^{\dagger}, \mathbf{Z}_{n}\right) S_{m n}$. For the systems with simple potentials, the ordered Hamiltonian can be calculated by using the ordered operator method $[15,16]$. For the systems with complicated potentials, such as the Coulomb potential, the ordered Hamiltonian has to be calculated by the direct integral method [14,18]. Obviously, $\langle H\rangle_{n}=\left\langle\psi_{n}|H| \psi_{n}\right\rangle=$ $H_{\text {ord }}\left(\mathbf{Z}_{n}^{\dagger}, \mathbf{Z}_{n}\right)$.

To derive the motion equation for the CSETs, the expansion coefficient $c_{n}(t)$ is rewritten as [17]

$$
c_{n}(t)=\eta_{n} \exp \left[i A_{n}(t)\right]
$$

where $\eta_{n}$ is a time-independent parameter and $A_{n}(t)$ is the "classical" action given by

$$
A_{n}(t)=\int_{0}^{t}\left[\frac{i \hbar}{2}\left(\mathbf{Z}_{n}^{\dagger} \cdot \dot{\mathbf{Z}}_{n}-\dot{\mathbf{Z}}_{n}^{\dagger} \cdot \mathbf{Z}_{n}\right)-\langle H\rangle_{n}\right] d t^{\prime} .
$$

From the quantum variational principle [17], the Lagrange equations of motion for the Lagrangian $\mathcal{L}$ is given by

$$
\frac{\partial \mathcal{L}}{\partial \mathbf{Z}_{n}^{\dagger}}-\frac{d}{d t} \frac{\partial \mathcal{L}}{\partial \dot{\mathbf{Z}}_{n}^{\dagger}}=0 .
$$

Substituting Eq. (7) into Eq. (6) and applying Eq. (6) to Eq. (9), one obtains a group of generalized Ehrenfest equations for the most probable CSETs:

$$
\frac{d \mathbf{Z}_{n}}{d t}=-\frac{i}{\hbar} \frac{\partial\langle H\rangle_{n}}{\partial \mathbf{Z}_{n}^{*}} .
$$

Since $\langle H\rangle_{n}=H_{\text {ord }}\left(\mathbf{Z}_{n}^{\dagger}, \mathbf{Z}_{n}\right)$ retains quantum effects, such as electron dynamic exchange, etc., the CSETs are quantum trajectories.

The equations given above offer a procedure for the study of dynamic processes of a system with a large number of particles. This procedure is very similar to that proposed in Ref. [17]. However, this procedure strongly depends on the initial wave function of the system since the electrons and nuclei are described by the wave functions. For the systems with a large number of particles such as polyatomic molecules, it is difficult to accurately calculate the initial states and propagate the wave functions. In the following we propose an alternative but more feasible method. In this method, instead of the wave functions, the electrons and nuclei are characterized by the CSETs in the phase space. The dynamic processes related to the electrons and nuclei are identified by the properties of the CSETs and the transition probability of a dynamic process is computed from the number of CSETs in this process.

\section{Husimi distribution and transition probability of a dynamic process}

For the $N_{p}$-particle system described by the wave function $|\Psi(t)\rangle$, the Husimi distribution in the phase space spanned by the CSs $\left\{\left|\chi_{n}(j)\right\rangle, j=1,2, \ldots, N_{p}\right\}$ is defined by $[19,20]$

$$
\rho_{H}\left(\mathbf{Z}_{n}\right)=\left|\left\langle\chi_{n}\left(N_{p}\right) \cdots \chi_{n}(2) \chi_{n}(1) \mid \Psi(t)\right\rangle\right|^{2} .
$$

It represents the probability density in the phase space for the $N_{p}$ particles to occupy $N_{p}$ volumes of magnitude $(2 \pi \hbar)^{3}$ at the trajectories $\mathbf{Z}_{n}$. From Eqs. (1), (5), and (11), for the system with a very large number of particles, the population probability density on the $n$th TDBS can be computed by

$$
\left|c_{n}(t)\right|^{2}=\left|\sum_{n^{\prime}=1}^{N_{t}} S_{n n^{\prime}} c_{n^{\prime}}(t)\right|^{2}=\rho_{H}\left(\mathbf{Z}_{n}\right) .
$$

It indicates that the population probability density on the $n$th TDBS is equal to the probability density of the $N_{p}$ particles at the trajectories $\mathbf{Z}_{n}$ in the phase space. Furthermore, in the $n$th TDBS, the expectation values of position $\left\langle\mathbf{x}_{j}\right\rangle_{n}$ and momentum $\left\langle\mathbf{p}_{\mathbf{x}_{j}}\right\rangle_{n}$ of the $j$ th particle are equal to $\mathbf{q}_{j n}$ and $\mathbf{p}_{j n}$, respectively. Thus a particle in the real space can be represented uniquely by a CSET in the phase space. Based on these correspondences, we propose to calculate the transition probability of a dynamic process by the percentage of the CSETs in this process. For example, the ionization probability of the $n$th TDBS is proportional to the number of electrons whose $\left|\mathbf{q}_{j n}\right|^{\prime}$ s are greater than the presupposed critical distance of ionization in this TDBS. The total ionization probability is the weighted sum of the ionization probability by $\left|c_{n}(t)\right|^{2}$ over all the TDBSs. Note that the population probability $\left|c_{n}(t)\right|^{2}=\left|\eta_{n}\right|^{2}$ is time independent for the proposed method from Eq. (7). However, each TDBS can contain various processes simultaneously depending on the properties of $\mathbf{Z}_{n}$. During the evolution of the system, the trajectories $\mathbf{Z}_{n}$ in a TDBS vary with time and thus the dynamic processes also change with time in this TDBS. For instance, a bound-state electron at this moment may become a photoelectron at the next moment. 
Conventionally, the initial state of the system is used to determine the coefficient $\eta_{n}$; the TDBSs are constructed first and then the value of $\eta_{n}$ is optimized to reproduce the initial state. However, from Eq. (1), the initial state can also be reproduced by adjusting the TDBSs after the distribution of $\eta_{n}$ is given. Although the choice of $\eta_{n}$ is flexible to some extent, a suitable choice with reasonably physical consideration will make the calculation simple and more accurate. To determine $\eta_{n}$ and initial CSETs, we make use of the initial state probability distribution and initial state energy of the system. More specifically, we first generate a set of CSETs and CSs using the Monte Carlo sampling technique together with the initial state probability distribution (using Gaussian distribution as an approximate initial state probability distribution for the complicated system whose initial state cannot be exactly known), then we construct the TDBSs and calculate the expectation value $\langle H\rangle_{n}$ using the CSs, and finally we pick up the CSETs by which the expectation value $\langle H\rangle_{n}$ is equal to or close to the energy of the initial state as the initial CSETs. Based on the correspondence principle, in the limit of a large number of particles, the states of the system with the same energy have the same population probability. The values of $\left|\eta_{n}\right|^{2}$ for different TDBSs with similar energies are close to each other in the system with a large number of particles. In the proposed approach, the values of $\left|\eta_{n}\right|^{2}$ are supposed to be the same for all the TDBSs. The basic equation of motion of the approach is the generalized Ehrenfest equations given by Eq. (10) for the CSETs. Once the initial CSETs are given, the CSETs at any time are obtained by solving Eq. (10). The dynamic processes can be identified by the properties of the CSETs and the transition probability of a process can be computed by the number of the CSETs in this process.

\section{RESULTS AND DISCUSSION}

\section{A. Dynamic processes of $\mathbf{H}_{2}$ in laser fields}

The proposed approach is applied to the study of full dynamics of $\mathrm{H}_{2}$ in laser fields for the case that the internuclear axis is parallel to the laser fields. In the laser field, if nuclear motions are taken into account, the Hamiltonian of $\mathrm{H}_{2}$ is given by

$$
\begin{aligned}
H\left(\mathbf{r}_{1}, \mathbf{r}_{2} ; \mathbf{R}_{1}, \mathbf{R}_{2} ; t\right) & =\sum_{j=1}^{2} \frac{\mathbf{p}_{j}^{2}}{2 m_{j}}+\frac{e^{2}}{\left|\mathbf{r}_{1}-\mathbf{r}_{2}\right|}+\sum_{\mu=1}^{2} \frac{\mathbf{P}_{\mu}^{2}}{2 M_{\mu}}+\frac{e^{2}}{\left|\mathbf{R}_{1}-\mathbf{R}_{2}\right|} \\
& -\sum_{j=1}^{2} \sum_{\mu=1}^{2} \frac{e^{2}}{\left|\mathbf{r}_{j}-\mathbf{R}_{\mu}\right|}+e \varepsilon(t) \cdot\left(\sum_{\mu=1}^{2} \mathbf{R}_{\mu}-\sum_{j=1}^{2} \mathbf{r}_{j}\right),
\end{aligned}
$$

where $e$ is the electronic charge, $\varepsilon(t)$ is the laser field, $m_{j}$ $\left(M_{\mu}\right), \mathbf{r}_{j}\left(\mathbf{R}_{\mu}\right)$, and $\mathbf{p}_{j}\left(\mathbf{P}_{\mu}\right)$ are the mass, position, and momentum of the $j$ th electron (the $\mu$ th nucleus), respectively. To acquire $\langle H\rangle_{n}$, we calculate the expectation values of kinetic energies and interaction energies with laser fields using the ordered operator method $[15,16]$ and the expectation values of Coulombic potentials employing the direct integral method
$[14,18]$. The CSETs of electrons and nuclei are computed by solving Eq. (10) with the fourth-order Runge-Kutta scheme. The dynamic properties of each electron or nucleus, such as the position, energy, and motion direction, etc., can be extracted from the CSETs.

The dynamic processes of $\mathrm{H}_{2}$ in intense laser fields, which include electronic and nuclear excitation, single-electron ionization, double-electron ionization, dissociation, dissociative electron ionization, dissociative electron attachment, and double-electron ionization Coulomb explosion, can be identified from relative positions of electrons and nuclei by the following conditions. Assume $r_{I}$ and $R_{D}$ are the critical distances of electron ionization and nuclear dissociation, respectively. If the internuclear distance is less than $R_{D}$, then the process is the electronic and nuclear excitation when the distances of the two electrons to at least one of the nuclei are less than $r_{I}$, the single-electron ionization when the distances of one and only one electron to both nuclei are larger than $r_{I}$, and the double-electron ionization when the distances of the two electrons to both nuclei are larger than $r_{I}$. If the internuclear distance is larger than $R_{D}$, then the process is the dissociation when the distances of the first electron to one nucleus and the second electron to another nucleus are less than $r_{I}$, the dissociative electron ionization when the distances of one electron to both the nuclei are larger than $r_{I}$ and the distance of another electron to one of the nuclei is less than $r_{I}$, the dissociative electron attachment when the distances of the two electrons to one nucleus are less than $r_{I}$, and the doubleelectron ionization Coulomb explosion when the distances of the two electrons to any of the two nuclei are larger than $r_{I}$. To avoid overlap and overslaugh of the dynamic processes we take $R_{D}=2 r_{I}$. To justify the conditions proposed above, we also perform calculations employing energy criteria. It is shown that when $R_{D}$ and $r_{I}$ are sufficiently large the results obtained from the above conditions are insensible to the values of $R_{D}$ as well as $r_{I}$ and are in fairly good agreement with those from the energy criteria, demonstrating that the conditions are reasonable.

The transition probability of one process can be calculated from the number of CSETs in this process. For example, for the single-electron ionization, the SDIP $d^{2} P(E, \theta, \phi) / d E d \Omega$, where $d \Omega=\sin \theta d \theta d \phi$, can be estimated from the number of photoelectrons within the solid angle $\Delta \Omega$ on the direction of $(\theta, \phi)$ and energy interval $\triangle E$ at the energy $E$ at the end of the laser pulse. For axially symmetric systems, $d \Omega(\theta)=$ $2 \pi \sin \theta d \theta$, which only depends on $\theta$.

To obtain converged results, we employ up to 500000 trajectories for every electron and nucleus in the calculation. To determine the initial electron trajectories, we first randomly generate a group of trial trajectories $\mathbf{Z}_{n}$ and CSs using the Monte Carlo sampling technique with a Gaussian distribution, then calculate the expectation values $\langle H\rangle_{n}=H_{\text {ord }}\left(\mathbf{Z}_{n}^{\dagger}, \mathbf{Z}_{n}\right)$ using these trajectories, and finally pick up the trajectories $\mathbf{Z}_{n}$ whose expectation values $\langle H\rangle_{n}$ are close to those of lower bound states as the initial electron CSETs [14]. Since the mass of nucleus is much larger than the electron's (about 1840 times the electron's), we assume that initially the nuclei are symmetrically located on the $z$ axis with equilibrium distance and the momentum of each nucleus being zero. The laser fields are depicted by $\varepsilon(t)=\varepsilon_{0} f(t) \sin (\omega t+\alpha)$, where $\varepsilon_{0}, \omega, \alpha$, and 

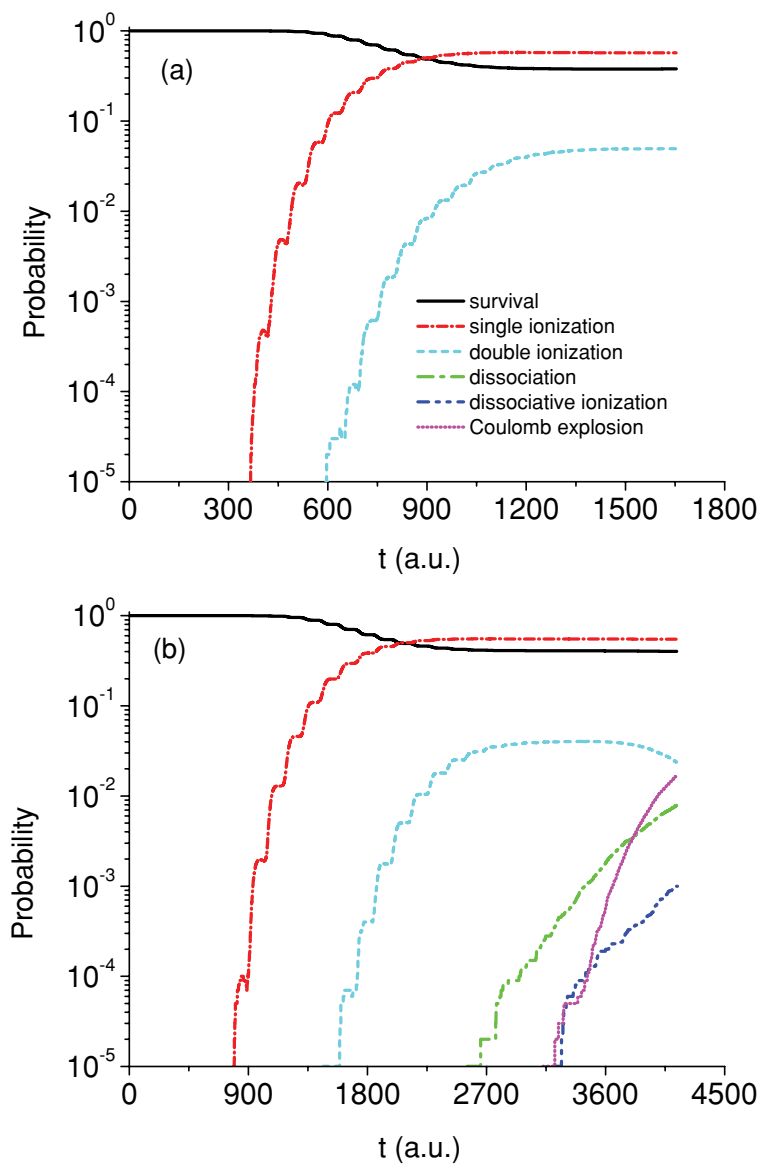

FIG. 1. (Color online) Evolution of transition probabilities of dynamic processes for $\mathrm{H}_{2}$ in the laser fields with $I_{0}=500 \mathrm{TW} \mathrm{cm} \mathrm{cm}^{-2}$ but $\lambda=800 \mathrm{~nm}$ (a) and $\lambda=2000 \mathrm{~nm}$ (b).

$f(t)$ are the amplitude, angular frequency, phase, and envelope shape factor of the laser fields, respectively. In the calculations given below, sine square pulses with duration of 15 optical cycles are used for intense laser fields and ramp-up-constant pulses for the calculation of photoionization cross sections in weak laser fields. In the calculation, all the dynamic processes are included and the critical distances used for the ionization and dissociative processes are $r_{I}=20$ a.u. and $R_{D}=40$ a.u.

In Fig. 1 we show evolution of transition probabilities of dynamic processes for $\mathrm{H}_{2}$ in intense laser fields with intensity $I_{0}=500 \mathrm{TW} \mathrm{cm}^{-2}$ but different wavelength $\lambda$. In the case of shorter wavelength with $\lambda=800 \mathrm{~nm}$ (also shorter laser duration) in Fig. 1(a), only single ionization and double ionization are induced. However, in the case of longer wavelength with $\lambda=2000 \mathrm{~nm}$ (also longer laser duration) in Fig. 1(b), the dissociation, dissociative ionization, and double-ionization Coulomb explosion also take place apart from the single ionization and double ionization. Because the nucleus is much heavier than the electron, the dynamic processes related to the nucleus always come out later than those related to the electron. In both cases, the dominant and secondarily dominant processes are the single ionization and double ionization apart from the (survival) excitation. The probability of the double ionization is less than that of the single ionization by at least one order of magnitude. The probabilities for all the other processes, such as dissociation and dissociative ionization, etc., are even smaller than the double-ionization probability. Thus we will focus our study on the single-ionization process in this work.

\section{B. Photoionization cross sections of $\mathbf{H}_{2}$}

For justification, the proposed approach is applied to the calculation of photoionization cross sections of $\mathrm{H}_{2}$ in weak laser fields. The laser pulses are ramped up linearly over 5 optical cycles and then kept constant thereafter. In the laser field that is not very strong, the norm of the wave function of bound states decreases exponentially as $\exp (-R t)$ during the constant amplitude of the laser, where $R$ is the ionization rate [21]. The ionization probability increases with time as $1-\exp (-R t)$ if the ionization is dominant. In the weak field limit, the ionization probability increases linearly with time as $R t$. From the evolution of the ionization probability obtained, the ionization rate $R$ is estimated and the photoionization cross sections $\sigma$ are computed by

$$
\sigma(\omega)=\frac{\hbar \omega R}{I_{0}}
$$

In the laser fields that are not very strong, the ionization rate $R \sim I_{0}^{N_{I}}$, where $N_{I}$ is the minimum number of photons to ionize $\mathrm{H}_{2}$. For the case considered here, the photon energy $\hbar \omega$ is greater than the ionization energy of $\mathrm{H}_{2}$ and $N_{I}=1$. The cross sections are independent of the laser field strength and polarization direction, and thus the orientation of $\mathrm{H}_{2}$ in the laser fields. In Fig. 2, we plot the calculated photoionization cross sections of $\mathrm{H}_{2}$ in the case where the laser fields are polarized along the internuclear axis together with the experimental data [22-24] and other theoretical results [25,26]. Since the ionization rate is estimated from the time evolution of the ionization probability the laser fields used in the calculation should not be very weak [21], depending on the laser

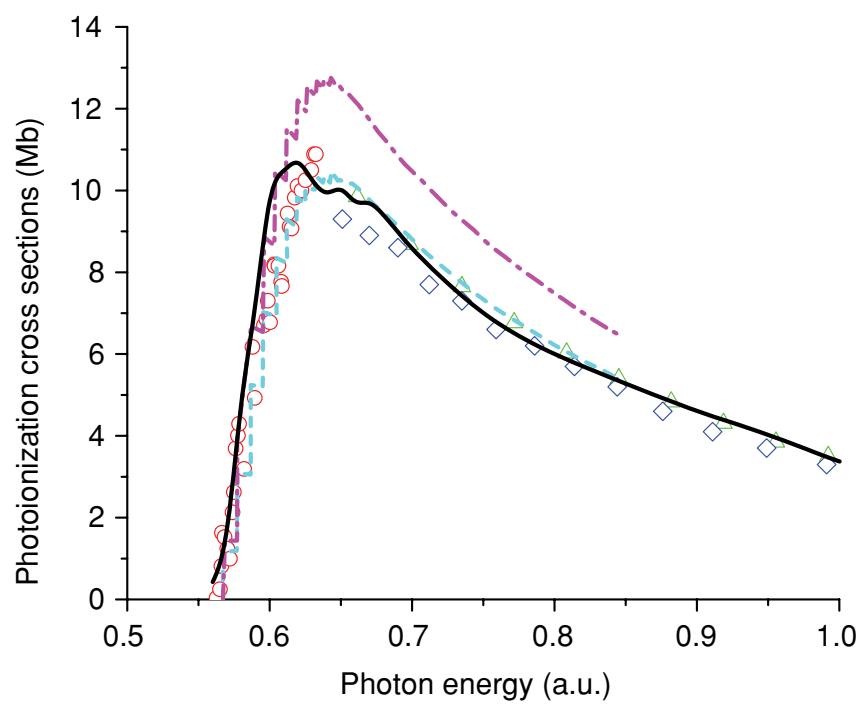

FIG. 2. (Color online) Photoionization cross section of $\mathrm{H}_{2}$ in weak laser fields. The solid line, the dashed line [25], and the dash-dotted line [26] are the theoretical results of the proposed approach in this work, the quantum-defect method, and the explicit Hilbert-space techniques, respectively, and the open circles [24], open triangles [23], and open diamonds [22] are the experimental data. 


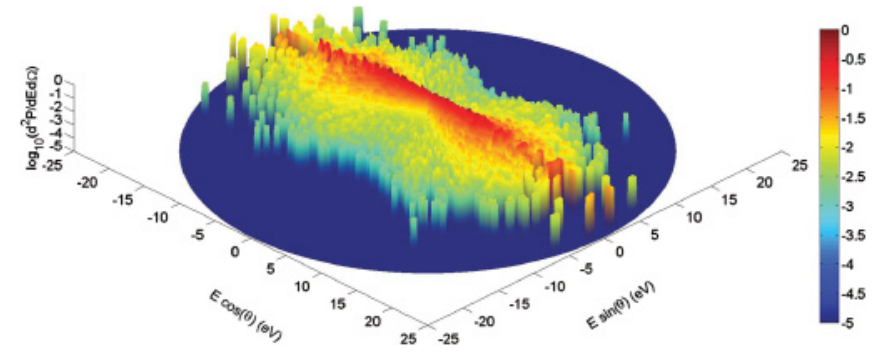

FIG. 3. (Color online) Energy-angular distribution of the normalized SDIP of $\mathrm{H}_{2}$ in the laser field with $\lambda=660 \mathrm{~nm}$ and $I_{0}=150 \mathrm{TW} \mathrm{cm}^{-2}$.

frequency. In our calculation the laser intensities range from $1 \times 10^{10}$ to $5 \times 10^{12} \mathrm{~W} / \mathrm{cm}^{2}$. It is shown that our calculated results are close to the theoretical results of the quantum-defect method [25] in the given energy range but smaller than those of explicit Hilbert-space techniques [26] in the highenergy region. Our results are also in good agreement with the experimental results [22-24].This demonstrates that the proposed approach can be used to describe the photoionization process of $\mathrm{H}_{2}$ in the laser fields.

\section{Energy-angular distribution of photoelectrons}

To investigate the energy-angular distribution of photoelectrons, the proposed approach is applied to calculate the SDIP of $\mathrm{H}_{2}$ in intense laser fields. For the sine square laser pulse with $I_{0}=150 \mathrm{TW} \mathrm{cm}^{-2}$ and $\lambda=660 \mathrm{~nm}$, the normalized SDIP on a plane through the internuclear axis is shown in Fig. 3 in the polar coordinates $(E, \theta)$, where $E$ is the photoelectron energy and $\theta$ is the angle of the photoelectron momentum with respect to the laser field. Due to cylindrical symmetry, the SDIP on the half plane of $180^{\circ} \leqslant \theta \leqslant 360^{\circ}$ is the mirror of that on the half plane of $0^{\circ} \leqslant \theta \leqslant 180^{\circ}$. The distribution of SDIP is a dumbbell shape with distinct boundaries on both $E$ and $\theta$ directions.

To see the details of the photoelectron distribution, we plot the PESa on different directions in Figs. 4(a)-4(d). For the laser field used in this calculation, $\gamma=1.124$ and the main contribution to the photoionization comes from the
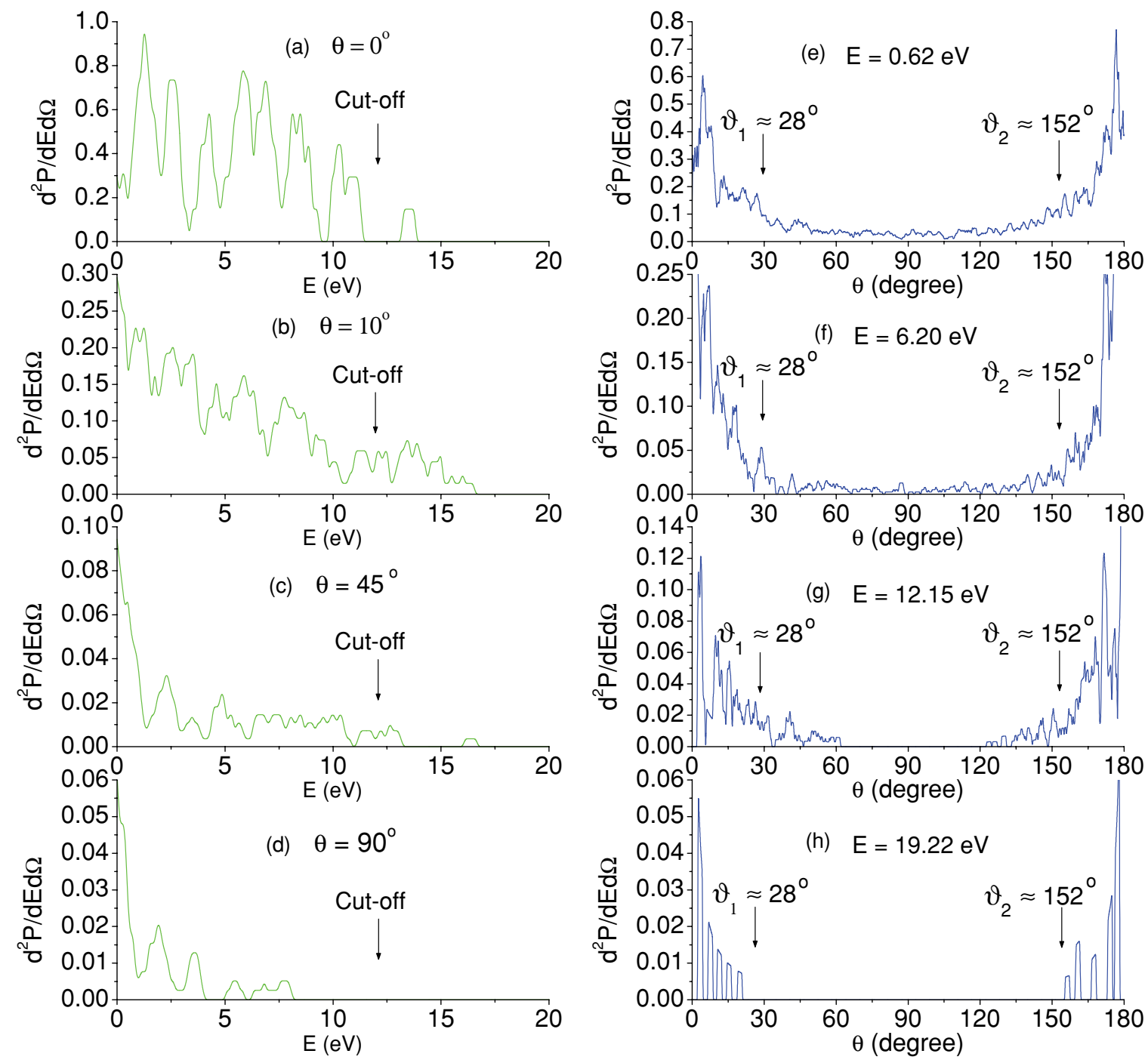

FIG. 4. (Color online) Photoelectron distribution of $\mathrm{H}_{2}$ in the laser field with $\lambda=660 \mathrm{~nm}$ and $I_{0}=150 \mathrm{TW} \mathrm{cm}^{-2}$. (a)-(d) PESa on different angular direction, and (e)-(h) PADs for different photoelectron energy. 

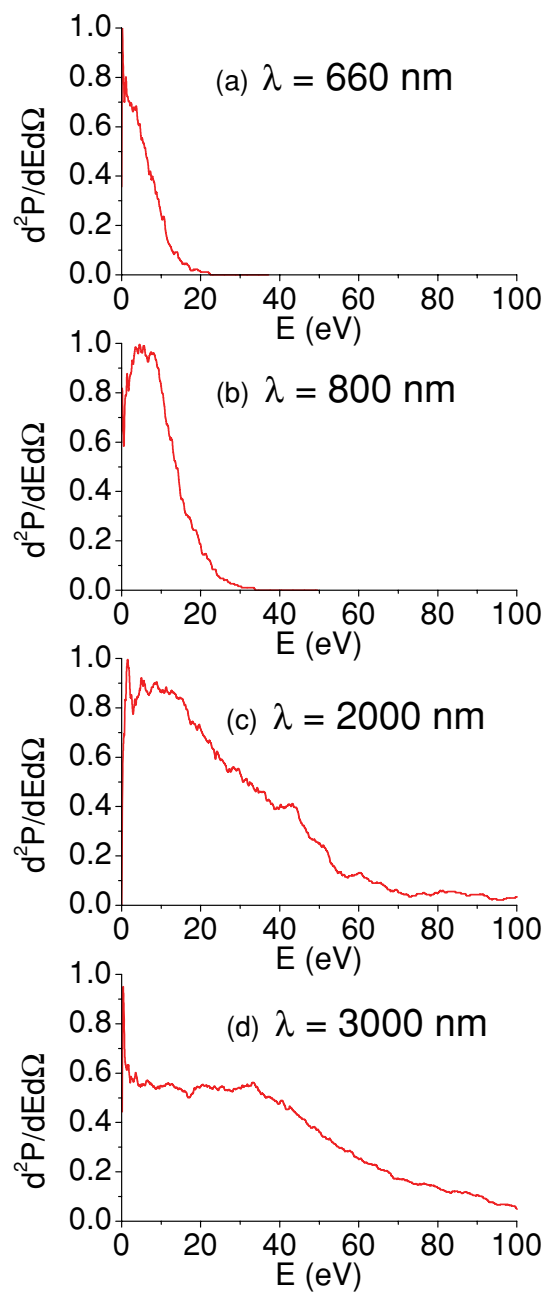
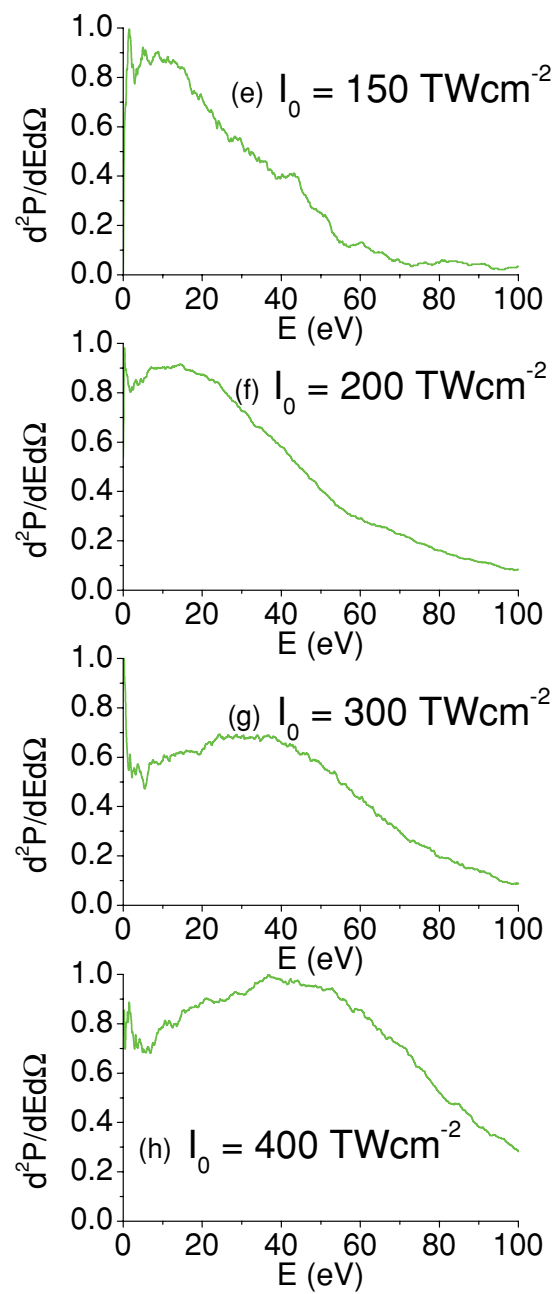
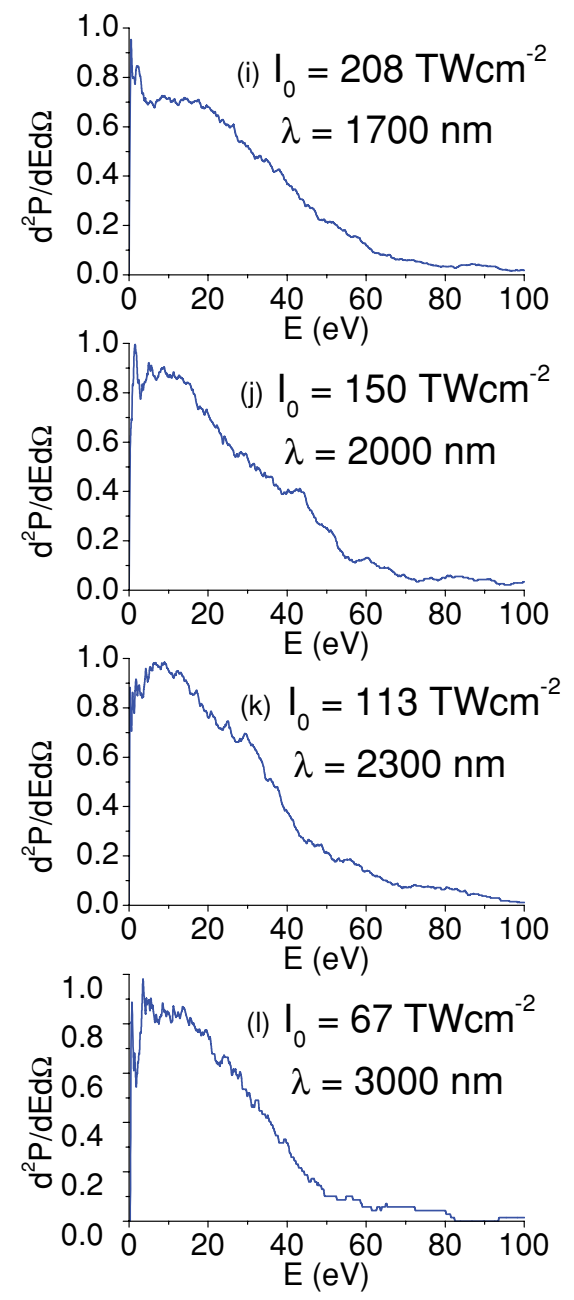

FIG. 5. (Color online) Normalized energy-averaged SDIPs on the direction of $\theta=6^{\circ}$ for $\mathrm{H}_{2}$ in various laser fields: (a)-(d) for the laser fields with $I_{0}=150 \mathrm{TW} \mathrm{cm}{ }^{-2}$ but different $\lambda$; (e)-(h) for the laser fields with $\lambda=2000 \mathrm{~nm}$ but different $I_{0}$; and (i)-(1) for the laser fields with different $\lambda$ and $I_{0}$ but the same $\gamma=0.371$.

multiphoton above-threshold ionization (ATI) although the tunneling also contributes [27]. The PESa display the typical

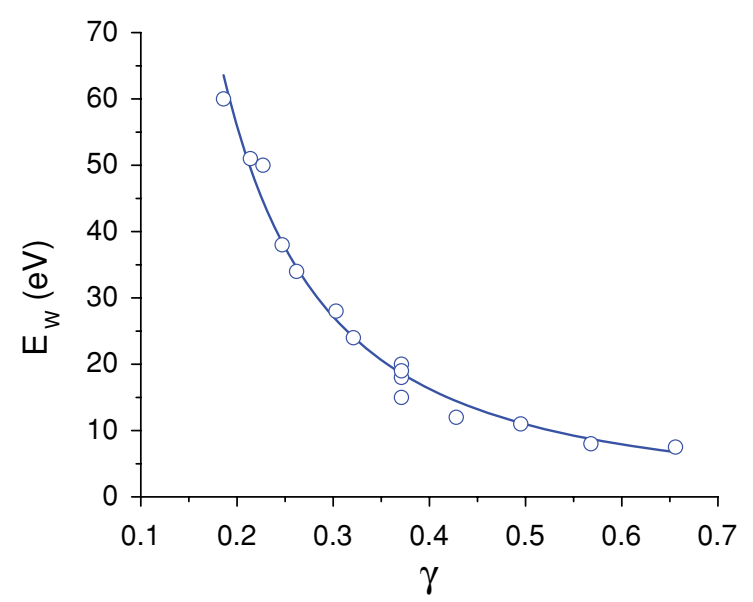

FIG. 6. (Color online) $E_{W}$ versus $\gamma$. The open circles are the numerical results and the solid line is the fitting result to $\gamma^{-\alpha}$ with $\alpha \approx 1.77$.
ATI characters: fast decay, plateau, and cutoff. But the details of the PESa, for example, peak, and cutoff positions change with $\theta$. On the direction of small $\theta$, such as $\theta=0$, the PESa have distinct peaks that are well separated by an approximately equal (photon) energy. On the direction with large $\theta$, the peaks become blurred. The PESa extend beyond the classical cutoff $2 U_{p}=12.20 \mathrm{eV}$ when $\theta \leqslant 50^{\circ}$, spread up to the classical cutoff when $50^{\circ}<\theta \leqslant 70^{\circ}$, and end before the classical cutoff when $70^{\circ}<\theta \leqslant 90^{\circ}$.

In Figs. 4(e)-4(h), we show the PADs in different photoelectron energy regions. The PADs have peak structures over the angles and the peak structures change with the energy. Overall, the PADs decrease with $\theta$ when $\theta<90^{\circ}$, increase with $\theta$ when $\theta>90^{\circ}$, arrive at the maximum at $\theta=0$ and $180^{\circ}$, and the minimum occurs at $\theta=90^{\circ}$. In the regions of low and intermediate (plateau) energies, the photoelectrons distribute over all the angles, while in the regions of classical cutoff energy and beyond, the photoelectrons mainly distribute in small solid angles along the laser field. This conclusion is in good agreement with that predicted for hydrogen atom [28].

Figures 3 and 4(e)-4(h) show that the PADs have a "cutoff" angle just as the PESa have cutoff energy. The "cutoff" angle 

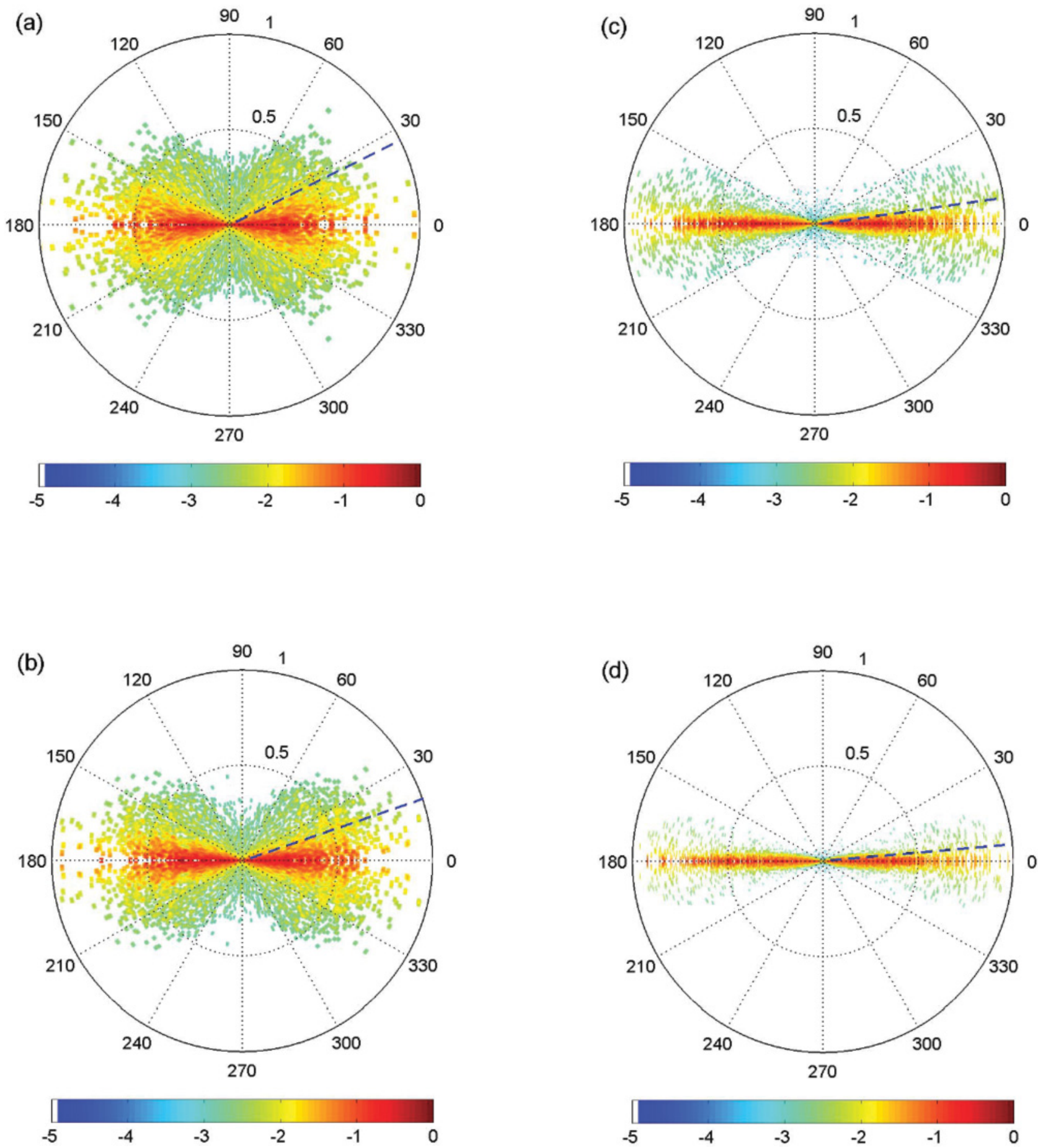

FIG. 7. (Color online) Color contour plots of the normalized SDIPs, $\log _{10}\left(\partial^{2} P / \partial E \partial \Omega\right)$, in the polar coordinates $(E, \theta)$ for $\mathrm{H}_{2}$ in the laser fields with $I_{0}=150 \mathrm{TW} \mathrm{cm}{ }^{-2}$ but different $\lambda$. (a) For $\lambda=660 \mathrm{~nm}$ and radial scale of $25 \mathrm{eV}$, (b) for $\lambda=800 \mathrm{~nm}$ and radial scale of $30 \mathrm{eV}$, (c) for $\lambda=1500 \mathrm{~nm}$ and radial scale of $70 \mathrm{eV}$, and (d) for $\lambda=2000 \mathrm{~nm}$ and radial scale of $120 \mathrm{eV}$. The dashed lines correspond to the maximum first "cutoff" angles.

is defined by an obvious change of the overall slope of PADs. The "cutoff" angles depend on photoelectron energy. For each energy, there are two "cutoff" angles $\theta_{1}<90^{\circ}$ and $\theta_{2}>90^{\circ}$, which we call the first and second "cutoff" angles, respectively. In the range of $\theta_{1} \leqslant \theta \leqslant \theta_{2}$, the PADs are much smaller than those in the other range. The larger the energy, the smaller the "cutoff" angle. The maximum "cutoff" angles of the
PADs in Fig. 3 are $\vartheta_{1} \approx 28^{\circ}$ and $\vartheta_{2} \approx 152^{\circ}$, as shown in Figs. 4(e)-4(h), where the PADs in different photoelectron energy regions - the low region (e), the plateau region (f), the cutoff region $(\mathrm{g})$, and the energy region beyond the cutoff (h) - are displayed in detail. Because of the cutoff energy in PESa and the "cutoff" angle in PADs, the photoelectrons distribute within a dumbbell. 

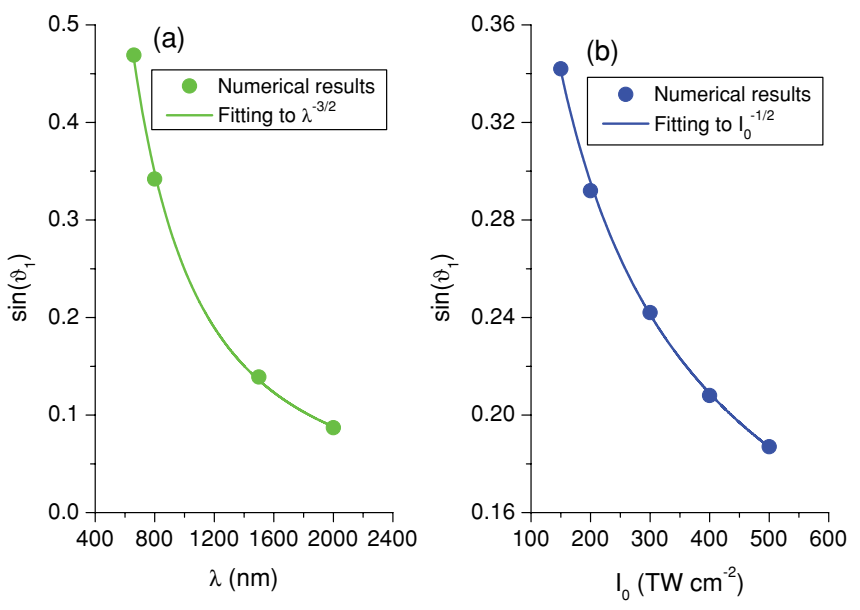

FIG. 8. (Color online) The maximum first "cutoff" angles $\vartheta_{1}$ for different laser fields: (a) $\sin \left(\vartheta_{1}\right)$ versus $\lambda$ for the laser fields of $I_{0}=150 \mathrm{TW} \mathrm{cm}{ }^{-2}$ and (b) $\sin \left(\vartheta_{1}\right)$ versus $I_{0}$ for the laser fields of $\lambda=800 \mathrm{~nm}$.

\section{Scaling law for the PESa}

To explore the LES features of PESa recently observed $[1,2]$, we study the PESa of $\mathrm{H}_{2}$ in the laser fields with different $\lambda$ and $I_{0}$. To make the PESa from both ATI and tunneling regimes comparable, we calculate energy-averaged SDIPs on the direction of $\theta=6^{\circ}$. The normalized SDIPs are shown in Figs. 5(a)-5(1) for the laser fields with $I_{0}=150 \mathrm{TW} \mathrm{cm}^{-2}$ but different $\lambda$, with $\lambda=2000 \mathrm{~nm}$ but different $I_{0}$, and with the same $\gamma(=0.371)$ but different $\lambda$ and $I_{0}$, respectively. When $\gamma \gtrsim 1$, there is only one peak near the ionization threshold on the PESa, as shown in Fig. 5(a). When $\gamma<1$, apart from the peak close to the threshold, there is a wide hump on the PESa, as shown in Figs. 5(b)-5(l), showing the LES [1,2].

To quantitatively study the LES, we introduce the width of the hump $E_{W}$ which is defined by a distinct change of the slope of PESa [1]. For a given $I_{0}, E_{W}$ increases with $\lambda$ and extends to high energy at long $\lambda$, as shown in Figs. 5(a)-5(d). These features are in accord with those observed recently for atoms [1,2]. For a given $\lambda, E_{W}$ increases with $I_{0}$, as shown in Figs. 5(e)-5(h). For a given $\gamma$, the PESa have very similar LES no matter what $I_{0}$ and $\lambda$ are, as shown in Figs. 5(i)-5(1). Thus the LES can be well characterized by $\gamma$. In Fig. 6 we plot the calculated $E_{W}$ versus $\gamma$ in open circles for the laser fields with different $\lambda$ and $I_{0}$. To find out the relation between $E_{W}$ and $\gamma$, we fit the numerical results with $\gamma^{-\alpha}$ and find that $\alpha \approx 1.77$. This scaling relation is in very good agreement with that obtained in the recent experiment for atoms [1], demonstrating it is universal for atoms and molecules.

\section{E. Scaling law for the PADs}

To investigate how the "cutoff" angles change with laser fields, we calculate the SDIPs for the laser fields with $I_{0}=150 \mathrm{TW} \mathrm{cm}{ }^{-2}$ but different $\lambda$. The results are plotted in Figs. 7(a)-7(d) with color contours in the polar coordinates $(E, \theta)$. Also plotted in the figures are the dashed lines corresponding to the maximum first "cutoff" angles $\vartheta_{1}$. The maximum first "cutoff" angles are about $28^{\circ}, 20^{\circ}, 8^{\circ}$, and $5^{\circ}$, respectively, for the SDIPs in Figs. 7(a)-7(d). Obviously, the PADs change greatly with $\lambda$. As $\lambda$ increases, $\vartheta_{1}$ decreases quickly and thus the PADs are squeezed. In Fig. 8(a) we plot $\sin \left(\vartheta_{1}\right)$ versus $\lambda$ in solid circles. We find that the relation between $\vartheta_{1}$ and $\lambda$ can be well represented by $\sin \left(\vartheta_{1}\right) \sim \lambda^{-3 / 2}$, as shown in Fig. 8(a) in the solid line. Thus $\sin \left(\vartheta_{1}\right)$ is inversely proportional to $\lambda^{3 / 2}$ and the photoelectrons will focus on the direction of the laser field in the case of the long-wavelength limit.

In order to investigate the relation between $\vartheta_{1}$ and $I_{0}$, we also calculate the SDIPs for the laser fields with $\lambda=800 \mathrm{~nm}$ but different $I_{0}$. In Fig. 8(b), we show $\sin \left(\vartheta_{1}\right)$ versus $I_{0}$ with the solid circles. We find that the relation between $\vartheta_{1}$ and $I_{0}$ can be well depicted by $\sin \left(\vartheta_{1}\right) \sim I_{0}^{-1 / 2}$, as shown in Fig. 8(b) in the solid line. Combining the results in Figs. 8(a) and 8(b), the scaling law of $\vartheta_{1}$ with respect to $\lambda$ and $I_{0}$ is given by

$$
\sin \left(\vartheta_{1}\right) \propto \lambda^{-3 / 2} I_{0}^{-1 / 2} .
$$

We have also checked this relation for the laser fields with different $\lambda$ and $I_{0}$ but the same $\lambda^{-3 / 2} I_{0}^{-1 / 2}$. The values of $\sin \left(\vartheta_{1}\right)$ do not change evidently in this case.

\section{CONCLUSIONS}

In summary, we have developed a more feasible CSET approach for the study of full dynamics of molecules interacting with intense laser fields. In this approach, the system is described by the coupled TDBSs of time-dependent CSs with pre-established coupling coefficients. The initial TDBSs are optimized by adjusting the positions and distribution density of the CSs to reproduce the initial state (probability density distribution) and energy of the system, and the TDBSs at any time are constructed by the time-dependent CSs whose positions are given by the CSETs-the CS trajectories determined by the coupled Ehrenfest equations. Through Husimi distribution as well as correspondence of the particle's position and momentum to those of the CS wave packet, evolution of a particle in real space is mapped to a CSET in phase space. The dynamic processes are distinguished by the properties of the CSETs and the transition probability of a process is calculated by the number of CSETs in this process. Since the CSETs are quantum trajectories, the proposed approach retains the quantum effects. The computational cost of the approach is linearly proportional to the dimension of the system and the larger the dimension of the system, the more accurate the approach will be.

We first computed the photoionization cross sections of $\mathrm{H}_{2}$ in the weak laser fields employing the proposed approach together with the Monte Carlo sampling technique. The results are in good agreement with the experimental data and other theoretical results. We then applied the proposed approach to explore the PESa of $\mathrm{H}_{2}$ in the intense laser fields with different $\lambda$ and $I_{0}$. The PESa show the typical characters of ATI spectra when $\gamma \gtrsim 1$. When $\gamma<1$, the PESa display LES and the LES extends to high energy with the increase of $\lambda$ and $I_{0}$. In particular, the scaling law of the LES with respect to $\gamma$ is in very good agreement with that observed recently for atoms, demonstrating the universality of the scaling law. We also applied the proposed approach to investigate the PADs of $\mathrm{H}_{2}$ in the intense laser fields with different $\lambda$ and $I_{0}$. We find that just 
as the PESa have cutoff energy, the PADs have "cutoff" angle. The maximum of the first "cutoff" angle decreases greatly with $\lambda$ and $I_{0}$. Thus the PADs are squeezed as $\lambda$ and $I_{0}$ increase, and the photoelectrons focus on the direction of the laser field in the limit of long $\lambda$ or/and high $I_{0}$. Finally, since the TDBSs tend to be orthogonal as the number of particles increases, the proposed approach is expected to provide a more effective, efficient, and accurate method for the systems with a large number of particles, in particular, for the polyatomic molecules in intense laser fields, where the existing quantum methods cannot be reached.

\section{ACKNOWLEDGMENTS}

Z.Z. is grateful to Dr. Dmitrii V. Shalashilin for helpful discussions on the coupled coherence state approach. This work is partially supported by the Chemical Sciences, Geosciences, and Biosciences Division of the Office of Basic Energy Sciences, Office of Science, Department of Energy, and by the National Science Foundation. We also acknowledge the partial support of the National Science Council of Taiwan (Grant No. 97-2112-M-002-003-MY3) and National Taiwan University (Grant No. 98R0066).
[1] C. I. Blaga, F. Catoire, P. Colosimo, G. G. Paulus, H. G. Muller, P. Agostini, and L. F. DiMauro, Nature Phys. 5, 335 (2009).

[2] W. Quan et al., Phys. Rev. Lett. 103, 093001 (2009).

[3] L. V. Keldysh, J. Exptl. Theoret. Phys. (USSR) 47, 1945 (1964) [Sov. Phys. JETP 20, 1307 (1965)].

[4] F. H. Faisal, J. Phys. B 6, L89 (1973).

[5] H. R. Reiss, Phys. Rev. A 22, 1786 (1980).

[6] X. Guan, X.-M. Tong, and S. I. Chu, Phys. Rev. A 73, 023403 (2006).

[7] C. W. McCurdy, D. A. Horner, T. N. Rescigno, and F. Martin, Phys. Rev. A 69, 032707 (2004).

[8] W. Vanroose, F. Martn, T. N. Rescigno, and C. W. McCurdy, Science 310, 1787 (2005).

[9] J. Colgan, M. S. Pindzola, and F. Robicheaux, Phys. Rev. Lett. 98, 153001 (2007).

[10] D. V. Shalashilin and M. S. Child, J. Chem. Phys. 121, 3563 (2004).

[11] M. H. Beck, A. Jckle, G. A. Worth, and H.-D. Meyer, Phys. Rep. 324, 1 (2000).

[12] G. A. Worth, H.-D. Meyer, H. Kppel, L. S. Cederbaum, and I. Burghardt, Int. Rev. Phys. Chem. 27, 569 (2008).

[13] I. Burghardt, K. Giri, and G. A. Worth, J. Chem. Phys. 129, 174104 (2008).

[14] D. V. Shalashilin, M. S. Child, and A. Kirrander, Chem. Phys. 347, 257 (2008).
[15] D. V. Shalashilin and M. S. Child, J. Chem. Phys. 113, 10028 (2000).

[16] D. V. Shalashilin and M. S. Child, Chem. Phys. 304, 103 (2004).

[17] D. V. Shalashilin and I. Burghardt, J. Chem. Phys. 129, 084104 (2008).

[18] D. V. Shalashilin and M. S. Child, J. Chem. Phys. 122, 224108 (2005).

[19] H. J. Korsch, C. Müller, and H. Wiescher, J. Phys. A: Math. Gen. 30, L677 (1997).

[20] L. E. Ballentine, Quantum Mechanics: A Modern Development (World Scientific Publishing, Singapore, 1998).

[21] K. C. Kulander, Phys. Rev. A 35, 445 (1987).

[22] L. C. Lee, R. W. Carlson, and D. L. Judge, J. Quant. Spectr. Rad. Trans. 16, 873 (1976).

[23] J. A. R. Samson and G. N. Haddad, J. Opt. Soc. Am. B 11, 277 (1994).

[24] M. Glass-Maujean, S. Klumpp, L. Werner, A. Ehresmann, and H. Schmoranzer, J. Chem. Phys. 126, 094306 (2007).

[25] G. Raseev and H. Le Rouzo, Phys. Rev. A 27, 268 (1983).

[26] M. R. Hermann and P. W. Langhoff, Phys. Rev. A 28, 1957 (1983).

[27] P. Eckle, A. N. Pfeiffer, C. Cirelli, A. Staudte, R. Drner, H. G. Muller, M. Bttiker, and U. Keller, Science 322, 1525 (2008).

[28] K. J. Schafer and K. C. Kulander, Phys. Rev. A 45, 8026 (1992). 